Received: 03.03.2021

Revised: 01.04.2021

Accepted: 23.04 .2021

DOI: $10.17804 / 2410-9908.2021 .2 .030-051$

\title{
EXACT SOLUTIONS OF THE NAVIER-STOKES EQUATIONS FOR DESCRIBING AN ISOBARIC ONE-DIRECTIONAL VERTICAL VORTEX FLOW OF A FLUID
}

\author{
N. V. Burmasheva ${ }^{\text {a) }}$, E. Yu. Prosviryakov ${ }^{\text {b)* }}$ \\ Institute of Engineering Science, Ural Branch of the Russian Academy of Sciences, \\ 34 Komsomolskaya St., Ekaterinburg, 620049, Russian Federation \\ a) iD https://orcid.org/0000-0003-4711-1894 nat_burm@mail.ru \\ b) (iD https://orcid.org/0000-0002-2349-7801 evgen_pros@mail.ru \\ *Corresponding author. E-mail: evgen_pros@mail.ru \\ Address for correspondence: 34 Komsomolskaya St., Ekaterinburg, 620049, Russian Federation \\ Tel.: +7 (343) 37535 76; fax: +7 (343) 3745330
}

The article proposes a family of exact solutions to the Navier-Stokes equations for describing isobaric inhomogeneous unidirectional fluid motions. Due to the incompressibility equation, the velocity of the inhomogeneous Couette flow depends on two coordinates and time. The expression for the velocity field has a wide functional arbitrariness. This exact solution is obtained by the method of separation of variables, and both algebraic operations (additivity and multiplicativity) are used to substantiate the importance of modifying the classical Couette flow. The article contains extensive bibliographic information that makes it possible to trace a change in the exact Couette solution for various areas of the hydrodynamics of a Newtonian incompressible fluid. The fluid flow is described by a polynomial depending on one variable (horizontal coordinate). The coefficients of the polynomial functionally depend on the second (vertical) coordinate and time; they are determined by a chain of the simplest homogeneous and inhomogeneous partial differential parabolic-type equations. The chain of equations is obtained by the method of undetermined coefficients after substituting the exact solution into the Navier-Stokes equation. An algorithm for integrating a system of ordinary differential equations for studying the steady motion of a viscous fluid is presented. In this case, all the functions defining velocity are polynomials. It is shown that the topology of the vorticity vector and shear stresses has a complex structure even without convective mixing (creeping flow).

Keywords: exact solution, Couette flow, Navier-Stokes equation, inhomogeneous unidirectional flow, method of separation of variables, shear stress.

\section{References}

1. Aristov S.N., Knyazev D.V., Polyanin A.D. Exact solutions of the Navier-Stokes equations with the linear dependence of velocity components on two space variables. Theoretical Foundations of Chemical Engineering, 2009, vol. 43, No. 5, pp. 642-662. DOI: 10.1134/S0040579509050066.

2. Drazin P.G., Riley N. The Navier-Stokes Equations: A classification of flows and exact solutions, Cambridge, Cambridge Univ. Press, 2006, 196 p.

3. Polyanin A.D., Zaitsev V.F. Handbook of nonlinear partial differential equations, Boca Raton, Chapman \& Hall / CRC Press, 2004, 840 p.

4. Yerin G.B. The Navier-Stokes equations of motion, Oxford, Clarendon, Laminar Boundary Layers, 1963, ed. L. Rosenhead, pp. 114-162. 
5. Dryden H.L., Murnaghan F.D., Bateman H. Report of the Committee on hydrodynamics. Bull. Natl. Res. Counc. (US), 1932, vol. 84, pp. 155-332.

6. Berker R. Sur quelques cas d'Integration des equations du mouvement d'un fuide visquex incomprcssible, Paris-Lille, Taffin-Lefort, 1936.

7. Berker R. Integration des equations du mouvement d'un fluide visqueux incompressible, Berlin, Springer-Verlag. Handbuch der Physik, ed. S. Flugge, 1963, VIII/2, 384 p.

8. Wang C.Y. Exact solution of the Navier-Stokes equations-the generalized Beltrami flows, review and extension. Acta Mech., 1990, vol. 81, pp. 69-74. DOI: 10.1007/BF01174556.

9. Wang C.Y. Exact solutions of the steady-state Navier-Stokes equations. Annu. Rev. Fluid Mech., 1991, vol. 23, pp. 159-177. DOI: 10.1146/annurev.fl.23.010191.001111.

10. Wang C.Y. Exact solutions of the unsteady Navier-Stokes equations. Appl. Mech. Rev. 1989, vol. 42 (11S), pp. 269-282. DOI: 10.1115/1.3152400.

11. Pukhnachev V.V. Symmetries in the Navier-Stokes equations. Uspekhi Mekhaniki, 2006, No. 1, pp. 6-76. (In Russian).

12. Couette M. Etudes sur le frottement des liquids. Ann. Chim. Phys., 1890, vol. 21, pp. 433-510.

13. Stokes G.G. On the effect of the internal friction of fluid on the motion of pendulums. Transactions of the Cambridge Philosophical Society, 1851, vol. 9, pp. 8-106.

14. Taylor G.I. Stability of a viscous fluid contained between two rotating cylinders. J. Phil. Trans. Royal Society A., 1923, vol. 223, No. 605-615, pp. 289-343. DOI: 10.1098/rsta.1923.0008.

15. Holodniok M., Kubíček M., Hlaváček V. Computation of the flow between two rotating coaxial disk: multiplicity of steady-state solutions. J. Fluid Mech., 1981, vol. 108, pp. 227-240. DOI: $10.1017 /$ S0022112081002097.

16. Aristov S.N., Gitman I.M. Viscous flow between two moving parallel disks: exact solutions and stability analysis. J. Fluid Mech., 2002, vol. 464, pp. 209-215. DOI: 10.1017/S0022112002001003.

17. Zhilenko D.Y., Krivonosova O.E. Transitions to chaos in the spherical Couette flow due to periodic variations in the rotation velocity of one of the boundaries. Fluid Dynamics, 2013, vol. 48, No. 4, pp. 452-460. DOI: 10.1134/S0015462813040042.

18. Zhilenko D., Krivonosova O., Gritsevich M., Read P. Wave number selection in the presence of noise: Experimental results. Chaos, 2018, vol. 28 (5), pp. 053110. DOI: 10.1063/1.5011349.

19. Zhilenko D.Y., Krivonosova O.E. Origination and evolution of turbulent flows in a rotating spherical layer. Technical Physics, 2010, vol. 55, No. 4, pp. 449-456. DOI: 10.1134/S1063784210040031.

20. Belyaev Yu.N., Monakhov A.A., Yavorskaya I.M. Stability of a spherical Couette flow in thick layers with rotation of the inner sphere. Izv. Akad. Nauk SSSR, Mekh. Zhidk. Gaza, 1978, No. 2, pp. 9-15. (In Russian).

21. Pukhnachev V.V., Pukhnacheva T.P. The Couette problem for a Kelvin-Voigt medium. J. Math. Sci., 2012, vol. 186, pp. 495-510. DOI: 10.1007/s10958-012-1003-0.

22. Zhuk V.I., Protsenko I.G. Asymptotic model for the evolution of perturbations in the plane Couette-Poiseuille flow. Doklady Mathematics, 2006, vol. 74, No. 3, pp. 896-900. DOI: $10.1134 / \mathrm{S} 1064562406060287$.

23. Gavrilenko S.L., Shil'ko S.V., Vasin R.A. Characteristics of a viscoplastic material in the Couette flow. Journal of Applied Mechanics and Technical Physics, 2002, vol. 43, No. 3, pp. 439-444. DOI: $10.1023 / \mathrm{A}: 1015378622918$.

24. Troshkin O.V. Nonlinear stability of Couette, Poiseuille, and Kolmogorov plane channel flows. Dokl. Math., 2012, vol. 85, pp. 181-185. DOI: 10.1134/S1064562412020068.

25. Rudyak V.Y., Isakov E.B. \& Bord E.G. Instability of plane Couette flow of two-phase liquids. Tech. Phys. Lett., 1998, vol. 24, pp. 199-200. DOI: 10.1134/1.1262051.

26. Shalybkov D.A. Hydrodynamic and hydromagnetic stability of the Couette flow. PhysicsUspekhi, 2009, vol. 52, No. 9, pp. 915-935. DOI: 10.3367/UFNe.0179.200909d.0971. 
27. Boronin S.A. Stability of the plane Couette flow of a disperse medium with a finite volume fraction of the particles. Fluid Dynamics, 2011, vol. 46, pp. 64-71. DOI: 10.1134/S0015462811010078.

28. Kudinov V.A. and Kudinov I.V. Calculation of Exact Analytic Solutions of Hyperbolic Equations of Motion in the Accelerated Couette Flow. Izv. Ross. Akad. Nauk. Energetika, 2012, No. 1, pp. 119-133. (In Russian).

29. Babkin V.A. Plane Turbulent Couette Flow. Journal of Engineering Physics and Thermophysics, 2003, vol. 76, pp. 1251-1254. DOI: 10.1023/B:JOEP.0000012026.19646.c6.

30. Abramyan A.K., Mirantsev L.V., Kuchmin A.Yu. Modeling of processes at Couette simple fluid flow in flat nano-scopic canal. Matem. Mod., 2012, vol. 24, Nos. 4, pp. 3-21. (In Russian).

31. Malyshev V. and Manita A. Stochastic Micromodel of the Couette Flow. Theor. Prob. Appl., 2009, vol. 53, no. 4. pp. 716-727. DOI: 10.1137/S0040585X97983924.

32. Georgievskii D.V. Generalized Joseph estimates of stability of plane shear flows with scalar nonlinearity. Bull. Russ. Acad. Sci. Phys., 2011, vol. 75, pp. 140-143. DOI: 10.3103/S1062873810121044.

33. Belyaeva N.A., Kuznetsov K.P. Analysis of a nonlinear dynamic model of the Couette flow for structured liquid in a flat gap. Vestn. Samar. Gos. Tekhn. Univ., Ser. Fiz.-Mat. Nauki [J. Samara State Tech. Univ., Ser. Phys. Math. Sci.], 2012, vol. 2 (27), pp. 85-92. (In Russian).

34. Neto C., Evans D., Bonaccurso E., Butt H.-J., Craig V.S.J. Boundary slip in Newtonian liquids: a review of experimental studies. Rep. Prog. Phys., 2005, vol. 68 (12), pp. 2859-2897. DOI: $10.1088 / 0034-4885 / 68 / 12 / \mathrm{R} 05$.

35. Beirão Da Veiga H. Regularity for Stokes and generalized Stokes systems under nonhomogeneous slip-type boundary conditions. Adv. Differential Equations, 2004, 9 (9-10), pp. 1079-1114.

36. Bocquet L., Charlaix E. Nanofluidics, from bulk to interfaces. Chem. Soc. Rev., 2010, vol. 39, pp. 1073-1095. DOI: 10.1039/b909366b.

37. Bouzigues C. I., Tabeling P., Bocquet L. Nanofluidics in the debye layer at hydrophilic and hydrophobic surfaces. Phys. Rev. Lett., 2008, vol. 101, pp. 114503.

38. Ng C.O., Wang C.Y. Apparent slip arising from Stokes shear flow over a bidimensional patterned surface. Microfluid Nanofluid, 2010, vol. 8, pp. 361-371. DOI: 10.1007/s 10404-009-0466-X.

39. Wang Y., Bhushan B. Boundary slip and nanobubble study in micro/nanofluidics using atomic force microscopy. Soft Matter., 2010, vol. 6, pp. 29-66. DOI: 10.1039/B917017K.

40. Schwarz K.G., Schwarz Y.A. Stability of Advective Flow in a Horizontal Incompressible Fluid Layer in the Presence of the Navier Slip Condition. Fluid Dyn., 2020, iss. 55, pp. 31-42. DOI: $10.1134 / \mathrm{S} 0015462820010115$.

41. Burmasheva N.V., Privalova V.V., Prosviryakov E.Y. Layered Marangoni convection with the Navier slip condition. Sãdhanā, 2021, vol. 46, pp. 55. DOI: 10.1007/s12046-021-01585-5.

42. Privalova V.V., Prosviryakov E.Yu. Nonlinear isobaric flow of a viscous incompressible fluid in a thin layer with permeable boundaries. Computational Continuum Mechanics, 2019, vol. 12, No. 2, pp. 230-242. DOI: 10.7242/1999-6691/2019.12.2.20. (In Russian).

43. Birikh R.V. Thermocapillary convection in a horizontal layer of liquid. J. Appl. Mech. Tech. Phys., 1966, No. 7, pp. 43-44. DOI: 10.1007/BF00914697.

44. Ostroumov G.A. Free convection under the condition of the internal problem. Washington, NACA Technical Memorandum 1407, National Advisory Committee for Aeronautics, 1958.

45. Smith M.K., Davis S.H. Instabilities of dynamic thermocapillary liquid layers. Part. 1. Convective instabilities. J. Fluid Mech, 1983, vol. 132, pp. 119-144. DOI: 10.1017/S0022112083001512.

46. Ortiz-Pérez A.S., Dávalos-Orozco L.A. Convection in a horizontal fluid layer under an inclined temperature gradient. Phys. Fluid, 2011, vol. 23, iss. 8, pp. 084107. DOI: 10.1063/1.3626009.

47. Burmasheva N.V., Prosviryakov E.Yu. Exact solution for stable convective concentration flows of a Couette type. Computational Continuum Mechanics, 2020, vol. 13, No. 3, pp. 337-349. DOI: 10.7242/1999-6691/2020.13.3.27. (In Russian). 
48. Aristov S.N., Shvarts K.G. Vikhrevye techeniya advektivnoy prirody vo vrashchayushchemsya sloe zhidkosti [Vortical Flows of the Advective Nature in a Rotating Fluid Layer]. Perm, Perm State Univ. Publ., 2006, 155 p. (In Russian).

49. Aristov S.N., Prosviryakov E.Y. A new class of exact solutions for three-dimensional thermal diffusion equations. Theoretical Foundations of Chemical Engineering, 2016, vol. 50, No. 3, pp. 286-293. DOI: 10.1134/S0040579516030027.

50. Burmasheva N.V., Prosviryakov E.Y. Thermocapillary convection of a vertical swirling liquid. Theoretical Foundations of Chemical Engineering, 2020, vol. 54, No. 1, pp. 230-239. DOI: $10.1134 / \mathrm{S} 0040579519060034$.

51. Birikh R.V., Pukhnachev V.V., Frolovskaya O.A. Convective flow in a horizontal channel with non-newtonian surface rheology under time-dependent longitudinal temperature gradient. Fluid Dynamics, 2015, vol. 50, No. 1, pp. 173-179. DOI: 10.1134/S0015462815010172.

52. Burmasheva N.V., Prosviryakov E.Yu. A large-scale layered stationary convection of a incompressible viscous fluid under the action of shear stresses at the upper boundary. Velocity field investigation. J. Samara State Tech. Univ., Ser. Phys. Math. Sci., 2017, vol. 21, No.1, pp. 180-196. DOI: 10.14498/vsgtu1527. (In Russian).

53. Burmasheva N.V., Prosviryakov E.Yu. A large-scale layered stationary convection of a incompressible viscous fluid under the action of shear stresses at the upper boundary. Temperature and pressure field investigation. J. Samara State Tech. Univ., Ser. Phys. Math. Sci., 2017, vol. 21, No. 4, pp. 736-751. DOI: 10.14498/vsgtu1568. (In Russian).

54. Burmasheva N.V., Prosviryakov E.Yu. Convective layered flows of a vertically whirling viscous incompressible fluid. Velocity field investigation. J. Samara State Tech. Univ., Ser. Phys. Math. Sci., 2019, vol. 23, No. 2, pp. 341-360. DOI: 10.14498/vsgtu1670.

55. Aristov S.N., Privalova V.V., Prosviryakov E.Yu. Stationary nonisothermal Couette flow. quadratic heating of the upper boundary of the fluid layer. Nelineynaya Dinamika, 2016, vol. 12, No. 2, pp. 167-178. DOI: 10.20537/nd1602001. (In Russian).

56. Burmasheva N.V., Prosviryakov E.Yu. On Marangoni shear convective flows of inhomogeneous viscous incompressible fluids in view of the Soret effect. Journal of King Saud University Science, 2020, vol. 32, iss. 8, pp. 3364-3371. DOI: 10.1016/j.jksus.2020.09.023.

57. Schwarz K.G. Plane-parallel advective flow in a horizontal incompressible fluid layer with rigid boundaries. Fluid Dynamics, 2019, vol. 49, No. 4, pp. 438-442. DOI: 10.1134/S0015462819110016.

58. Andreev V.K., Stepanova I.V. Unidirectional flows of binary mixtures within the framework of the Oberbeck-Boussinesq model. Fluid Dynamics, 2016, vol. 51, No. 2, pp. 136-147. DOI: 10.1134/S0015462816020022.

59. Andreev V.K., Stepanova I.V., Ostroumov-Birikh solution of convection equations with nonlinear buoyancy force. Appl. Math. Comput., 2014, vol. 228, pp. 59-67. DOI: 10.1016/j.amc.2013.11.002.

60. Bekezhanova V.B. Change of the types of instability of a steady two-layer flow in an inclined channel. Fluid Dynamics, 2011, vol. 46 (525). DOI: 10.1134/S001546281104003X.

61. Gorshkov A.V., Prosviryakov E.Y. Ekman convective layer flow of a viscous incompressible fluid. Izvestiya. Atmospheric and Oceanic Physics, 2018, vol. 54, No. 2, pp. 189-195. DOI: 10.1134/S0001433818020081. (In Russian).

62. Pukhnachev V.V. Non-stationary analogues of the Birikh solution. Izv. AltGU, 2011, Nos. 1-2 (69), pp. 62-69. (In Russian).

63. Shvarz K.G. Plane-parallel advective flow in a horizontal incompressible fluid layer with rigid boundaries. Fluid Dynamics, 2014, vol. 49, No. 4, pp. 438-442. DOI: 10.1134/S0015462814040036.

64. Andreev V.K. Resheniya Birikha uravneniy konvektsii i nekotoryye ego obobshcheniya [Birich's solutions of convection equations and some of its generalizations: preprint]. Krasnoyarsk, IVM SO RAN Publ., 2010, 24 p. (In Russian). 
65. Aristov S.N., Prosviryakov E.Y., Spevak L.F. Unsteady-state Bénard-Marangoni convection in layered viscous incompressible flows. Theoretical Foundations of Chemical Engineering, 2016, vol. 50, No. 2, pp. 132-141. DOI: 10.1134/S0040579516020019.

66. Aristov S.N., Prosviryakov E.Yu., Spevak L.F. Nonstationary laminar thermal and solutal Marangoni convection of a viscous fluid. Computational Continuum Mechanics, 2015, vol. 8, No. 4, pp. 445-456. DOI: 10.7242/1999-6691/2015.8.4.38. (In Russian).

67. Aristov S.N., Prosviryakov E.Yu. On laminar flows of planar free convection. Nelineynaya Dinamika, 2013, vol. 9, No. 4, pp. 651-657. (In Russian).

68. Gorshkov A.V., Prosviryakov E.Y. Layered B'enard-Marangoni convection during heat transfer according to the newton's law of cooling. Computer Research and Modeling, 2016, vol. 8, No. 6, pp. 927-940. (In Russian).

69. Gorshkov A.V., Prosviryakov E.Y. Analytic solutions of stationary complex convection describing a shear stress field of different signs. Trudy IMM UrO RAN, 2017, vol. 23, No. 2, pp. 32-41. DOI: 10.21538/0134-4889-2017-23-2-32-41. (In Russian).

70. Knyazev D.V. Two-dimensional flows of a viscous binary fluid between moving solid boundaries. Journal of Applied Mechanics and Technical Physics, 2011, vol. 52, No. 2, pp. 212-217. DOI: 10.1134/S0021894411020088.

71. Kompaniets L.A., Pitalskaya O.S. Exact solutions of Ekman's model for three-dimensional wind-induced flow of homogeneous fluid with geostrophic current. Computer Research and Modeling, 2009, vol. 1, No.1, pp. 57-66. DOI: 10.20537/2076-7633-2009-1-1-57-66. (In Russian).

72. Burmasheva N.V., Prosviryakov E.Yu. Exact solution of Navier-Stokes equations describing spatially inhomogeneous flows of a rotating fluid. Trudy IMM UrO RAN, 2020, vol. 26, No. 2, pp. 79-87. DOI: 10.21538/0134-4889-2020-26-2-79-87. (In Russian).

73. Burmasheva N.V., Prosviryakov E.Yu. A class of exact solutions for two-dimensional equations of geophysical hydrodynamics with two Coriolis parameters. The Bulletin of Irkutsk State University. Series «Mathematics», 2020, vol. 32, pp. 33-48. DOI: 10.26516/1997-7670.2020.32.33. (In Russian).

74. Burmasheva N.V., Prosviryakov E.Yu. An exact solution for describing the unidirectional Marangoni flow of a viscous incompressible fluid with the Navier boundary condition. Temperature field investigation. Diagnostics, Resource and Mechanics of materials and structures, 2020, iss. 1, pp. 6-23. DOI: 10.17804/2410-9908.2020.1.006-023. Available at: https://dream-journal.org/issues/2020-1/20201_278.html

75. Burmasheva N.V., Prosviryakov E.Yu. Exact solution for describing a unidirectional Marangoni flow of a viscous incompressible fluid with the Navier boundary condition. Pressure field investigation. Diagnostics, Resource and Mechanics of materials and structures, 2020, iss. 2, pp. 61-75, DOI: 10.17804/2410-9908.2020.2.061-075. Available at: https://dreamjournal.org/DREAM_Issue_2_2020_Burmasheva_N.V._et_al._061_075.pdf

76. Lin C.C. Note on a class of exact solutions in magneto-hydrodynamics. Arch. Rational Mech. Anal., 1957, vol. 1, pp. 391-395. DOI: 10.1007/BF00298016.

77. Sidorov A.F. Two classes of solutions of the fluid and gas mechanics equations and their connection to traveling wave theory. J. Appl. Mech. Tech. Phy., 1989, vol. 30, No. 2, pp. 197-203. DOI: $10.1007 / \mathrm{BF} 00852164$.

78. Aristov S.N. Eddy currents in thin liquid layers: Synopsis of a Dr. Phys. \& Math. Sci. Thesis, Vladivostok, 1990, 303 p. (In Russian).

79. Prosviryakov E.Y. New class of exact solutions of navier-stokes equations with exponential dependence of velocity on two spatial coordinates. Theoretical Foundations of Chemical Engineering, 2019, vol. 53, No. 1, pp. 107-114. DOI: 10.1134/S0040579518060088.

80. Aristov S.N., Prosviryakov E.Y. Large-scale flows of viscous incompressible vortical fluid. Russian Aeronautics, 2015, vol. 58, No. 4, pp. 413-418. DOI: 10.3103/S1068799815040091. 
81. Aristov S.N., Prosviryakov E.Y. Inhomogeneous Couette flow. Nelineynaya Dinamika, 2014, vol. 10, No. 2, pp. 177-182. DOI: 10.20537/nd1402004. (In Russian).

82. Privalova V.V., Prosviryakov E.Yu. Vortex flows of a viscous incompressible fluid at constant vertical velocity under perfect slip conditions. Diagnostics, Resource and Mechanics of materials and structures, 2019, iss. 2, pp. 57-70. DOI: 10.17804/2410-9908.2019.2.057-070. Available at: https://dream-journal.org/issues/2019-2/2019-2_249.html

83. Aristov S.N., Prosviryakov E.Y. Unsteady layered vortical fluid flows. Fluid Dynamics, 2016, vol. 51, No. 2, pp. 148-154. DOI: 10.1134/S0015462816020034.

84. Zubarev N.M., Prosviryakov E.Y. Exact solutions for layered three-dimensional nonstationary isobaric flows of a viscous incompressible fluid. Journal of Applied Mechanics and Technical Physics, 2019, vol. 60, No. 6, pp. 1031-1037. DOI: 10.1134/S0021894419060075.

85. Prosviryakov E.Yu. Exact solutions of three-dimensional potential and vortical Couette flows of a viscous incompressible fluid. Bulletin of the National Research Nuclear University MIFI, 2015, vol. 4, No. 6, pp. 501-506. DOI: 10.1134/S2304487X15060127. (In Russian).

86. Privalova V.V., Prosviryakov E.Yu., Simonov M.A. Nonlinear gradient flow of a vertical vortex fluid in a thin layer. Nelineynaya Dinamika, 2019, vol. 15, No. 3, pp. 271-283. DOI: 10.20537/nd190306. (In Russian).

87. Aristov S.N., Prosviryakov E.Y. Nonuniform convective Couette flow. Fluid Dynamics, 2016, vol. 51, No. 5, pp. 581-587. DOI: 10.7868/S0568528116050030. (In Russian).

88. Privalova V.V., Prosviryakov E.Yu. The effect of tangential boundary stresses on the convective unidirectional flow of viscous fluid layer under the heating the lower boundary condition. Diagnostics, Resource and Mechanics of materials and structures, 2019, iss. 4, pp. 44-55. DOI: 10.17804/2410-9908.2019.4.044-055. Available at: https://dream-journal.org/issues/2019-4/2019$4 \_262 . \mathrm{html}$

89. Privalova V.V., Prosviryakov E.Yu. Exact solutions for the convective creep Couette-Hiemenz flow with the linear temperature distribution on the upper border. Diagnostics, Resource and Mechanics of materials and structures, 2018, iss. 2, pp. 92-109. DOI: 10.17804/2410-9908.2018.2.092-109. Available at: https://dream-journal.org/DREAM_Issue_2_2018_Privalova_V.V._et_al._092_109.pdf

90. Aristov S.N., Privalova V.V., Prosviryakov E.Yu. Planar linear Benard-Rayleigh convection with quadratic heating of the upper boundary of a layer of a viscous incompressible fluid. Vestnik Kazanskogo gos. tekhn. Universiteta im. A.M. Tupoleva, 2015, vol. 71, No. 2, pp. 69-75. (In Russian).

91. Privalova V.V., Prosviryakov E.Yu. Stationary convective Couette flow with linear heating of the lower boundary of the liquid layer. Vestnik Kazanskogo gos. tekhn. Universiteta im. A.M. Tupoleva, 2015, vol. 71, No. 2, pp. 148-153. (In Russian).

92. Aristov S.N., Privalova V.V., Prosviryakov E.Y. Stationary nonisothermal Couette flow. Quadratic heating of the upper boundary of the fluid layer. Russian Journal of Nonlinear Dynamics, 2016, vol. 12, No. 2, pp. 167-178. DOI: 10.20537/nd1602001. (In Russian).

93. Aristov S.N., Prosviryakov E.Y. On one class of analytic solutions of the stationary axisymmetric convection Bénard-Maragoni viscous incompreeible fluid. Journal of Samara State Technical University, Ser. Physical and Mathematical Sciences, 2013, No. 3 (32), pp. 110-118. (In Russian).

94. Aristov S.N., Prosviryakov E.Y. Exact solutions of thermocapillary convection with localized heating of a flat layer of a viscous incompressible fluid. Vestnik Kazanskogo gos. tekhn. Universiteta im. A.M. Tupoleva, 2014, No. 3, pp. 7-12. (In Russian).

95. Andreev V.K., Cheremnykh E.N. 2D thermocapillary motion of three fluids in a flat channel. Journal of Siberian Federal University. Mathematics and Physics, 2016, vol. 9, iss. 4, pp. 404-415. DOI: 10.17516/1997-1397-2016-9-4-404-415. 
96. Andreev V.K., Cheremnykh E.N. The joint creeping motion of three viscid liquids in a plane layer: A priori estimates and convergence to steady flow. Journal of Applied and Industrial Mathematics, 2016, vol. 10, No. 1, pp. 7-20. DOI: 10.1134/S1990478916010026.

97. Andreev V.K., Sobachkina N.L. Dvizhenie binarnoy smesi v ploskikh i cilindricheskikh oblastyazkh [Movement of a binary mixture in flat and cylindrical regions]. Krasnoyarsk, SFU Publ., 2012, 187 p. (In Russian).

98. Hiemenz K. Die Grenzschicht an einem in den gleichförmigen Flüssigkeit-sstrom eingetauchten geraden Kreiszylinder. Dingler's Politech. J., 1911, vol. 326, pp. 321-324.

99. Riabouchinsky D. Quelques considerations sur les mouvements plans rotationnels d'un liquid. C. R. Hebdomadaires Acad. Sci., 1924, vol. 179, pp. 1133-1136.

100. Landau L.D., Lifshitz E.M. Fluid Mechanics: Volume 6 (Course of Theoretical Physics), 2nd ed., Elsevier, 558 p.

101. Polyanin A.D., Zhurov A.I. Metody razdeleniya peremennykh i tochnye resheniya nelineynykh uravneniy matematicheskoy fiziki [Methods of separation of variables and exact solutions of nonlinear equations of mathematical physics]. Moscow, Izd-vo IPMekh RAN Publ., 2020, 384 p. (In Russian).

102. Aristov S.N., Polyanin A.D. Exact solutions of unsteady three-dimensional Navier-Stokes equations. Doklady Physics, 2009, vol. 54, No. 7, pp. 316-321. DOI: 10.1134/S1028335809070039.

103. Polyanin A.D. Exact generalized separable solutions of the Navier-Stokes equations. Doklady RAN, 2001, vol. 380, No. 4, pp. 491-496. (In Russian).

104. Polyanin A.D. Methods of functional separation of variables and their application in mathematical physics. Matematicheskoe Modelirovanie i Chislennye Metody, 2019, No. 1, pp. 65-97. DOI: 10.18698/2309-3684-2019-1-6597. (In Russian).

105. Polyanin A.D., Aristov S.N. Systems of hydrodynamic type equations: exact solutions, transformations, and nonlinear stability. Doklady Physics, 2009, vol. 54, No. 9, pp. 429-434. DOI: 10.1134/S1028335809090079.

106. Polyanin A.D., Zhurov A.I. Functional separable solutions of two classes of nonlinear mathematical physics equations. Doklady AN, 2019, vol. 486, No. 3, pp. 287-291. DOI: 10.31857/S086956524863287-291. (In Russian).

107. Aristov S.N., Polyanin A.D. New classes of exact solutions and some transformations of the Navier-Stokes equations. Russian J. Math. Physics, 2010, vol. 17, No. 1, pp. 1-18. DOI: $10.1134 / \mathrm{S} 1061920810010012$.

108. Meleshko S.V. A particular class of partially invariant solutions of the Navier-Stokes equations. Nonlinear Dynamics, 2004, vol. 36, No. 1, pp. 47-68, DOI: 10.1023/B:NODY.0000034646.18621.73. 
Подана в журнал: 03.03.2021

УДК 517.958

DOI: $10.17804 / 2410-9908.2021 .2 .030-051$

\title{
ТОЧНЫЕ РЕШЕНИЯ УРАВНЕНИЙ НАВЬЕ-СТОКСА ДЛЯ ОПИСАНИЯ ИЗОБАРИЧЕСКОГО ОДНОНАПРАВЛЕННОГО ВЕРТИКАЛЬНО ЗАВИХРЕННОГО ТЕЧЕНИЯ ЖИДКОСТИ
}

\author{
Н. В. Бурмашева ${ }^{\text {a) }}$ Е. Ю. Просвиряков ${ }^{\text {б)* }}$ \\ Институт машиноведения УрО РАН, \\ 34, ул. Комсомольская, г. Екатеринбург, Российская Федерация \\ a) iD https://orcid.org/0000-0003-4711-1894 nat_burm@mail.ru \\ б) (iD https://orcid.org/0000-0002-2349-7801 ㅈevgen_pros@mail.ru \\ *Ответственный автор. Электронная почта: evgen_pros@mail.ru \\ Адрес для переписки: ул. Комсомольская, 34, Екатеринбург, Российская Федерация \\ Тел.: +7 (343) 375-35-76; факс: +7 (343) 374-53-30
}

В статье предложено семейство точных решений уравнений Навье-Стокса для описания изобарических неоднородных однонаправленных движений жидкости. Из-за уравнения несжимаемости скорость неоднородного течения Куэтта зависит от двух координат и времени. Приведенное выражение для поля скоростей обладает широким функциональным произволом. Данное точное решение получено методом разделения переменных, причем используются обе алгебраические операции (аддитивность и мультипликативность) для обоснования важности модификации классического течения Куэтта. В статье собрана значительная библиографическая информация, позволяющая проследить изменение точного решения Куэтта для различных областей гидродинамики ньютоновской несжимаемой жидкости. Течение жидкости представлено полиномом, зависящим от одной переменной (горизонтальной координаты). Коэффициенты полинома функционально зависят от второй (вертикальной) координаты и времени и определяются цепочкой простейших однородных и неоднородных уравнений в частных производных параболического типа. Цепочка уравнений получена методом неопределенных коэффициентов после подстановки точного решения в уравнение Навье-Стокса. Приведен алгоритм интегрирования системы обыкновенных дифференциальных уравнений для изучения установившегося движения вязкой жидкости. В этом случае все функции, определяющие скорость, являются полиномами, топология вектора завихренности и касательных напряжений даже без конвективного перемешивания (ползущее течение) имеет сложную структуру.

Ключевые слова: точное решение, течение Куэтта, уравнение Навье-Стокса, неоднородное однонаправленное течение, метод разделения переменных, касательное напряжение.

\section{1. Введение}

Проводя ретроспективный анализ научной литературы, можно сделать вывод, что одними из первых точных решений уравнений Навье-Стокса являлись формулы, описывающие изобарические потоки жидкости [1-11]. К таким решениям можно отнести однонаправленные течения Куэтта и две задачи Стокса [12;13]. Точное решение Куэтта описывает слоистое течение вязкой несжимаемой жидкости в бесконечном слое между одной или двумя подвижными границами [1-13]. Для первой и второй задач Стокса характерно изучение нестационарных потоков, индуцируемых различными возмущениями границ слоя жидкости [13]. Не будет преувеличением утверждение о том, что точные решения Куэтта и Стокса положи- 
ли начало теории точного интегрирования уравнений Навье-Стокса для несжимаемых жидкостей в различных силовых полях [1-13].

Течение Куэтта в цилиндрической области описывается точным решением КуэттаТейлора (течение жидкости между коаксиальными цилиндрами) [14-16]. Движение жидкости в сферической области так же, как точные решения Куэтта и Куэтта-Тейлора, имеет имя - сферическое течение Куэтта [17-20].

С теоретической точки зрения решение Куэтта востребовано в теории гидродинамической устойчивости [21-33]. Известно, что использование профиля Куэтта в качестве основного течения используется при изучении влияния различных типов возмущений (вторичных течений) на структуру изотермических гидродинамических потоков в бесконечно протяженных слоях жидкости, в областях с цилиндрической и сферической симметрией [21-33].

Течение Куэтта нашло свое применение в гидродинамике, протекающей на масштабах наномира и микрофлюидике [34-41]. Использование профиля Куэтта позволяет теоретически визуализировать длину скольжения при движении жидкости по гидрофобной поверхности в протяженной слое [34-42].

Применение структуры точного решения Куэтта в теории конвекции привело к первому классу точных решений уравнений Обербека-Буссинеска - семейству ОстроумоваБириха [43-46]. Обобщение точного решения для однонаправленных конвективных, диффузионных и термодиффузионных потоков вращающихся и невращающихся жидкостей приведено в библиографических источниках [47-76].

В сравнительно недавно опубликованных статьях было освещено, что в рамках класса Линя-Сидорова-Аристова [77-80] было построено обобщение течения типа Куэтта, индуцированное неоднородным распределением скоростей и касательных напряжений на границе жидкости и атмосферного воздуха [81-84]. Неоднородное распределение кинематических характеристик приводит к образованию вертикальной закрутки жидкости при отсутствии вращения [81-84]. Эти решения позволяют исследовать течения в экваториальной зоне Мирового океана в приближении $f$-плоскости.

Движение жидкости может быть индуцировано не только при помощи движения границ, но и посредством действия силовых полей. Интересные решения, обобщающие результат Куэтта, приведены в статьях [50; 85-87]. При рассмотрении ползущих плоских или трехмерных конвективных течений типа Куэтта в горизонтальных слоях с проницаемыми границами [88-92] или с учетом термокапиллярного эффекта Марангони [93-97] описывается семейством Хименца-Рябушинского [98; 99].

Учитывая важность использования классического точного решения Куэтта в теоретических и экспериментальных исследованиях, в статье осуществляется построение точных решений для описания однонаправленных изобарических и изотермических потоков жидкости с неоднородным распределением скорости и касательных напряжений на границах горизонтального слоя жидкости.

\section{2. Постановка задачи}

Изобарическое течение вязкой несжимаемой жидкости в бесконечном горизонтальном слое представлено следующей системой уравнений, записанной в векторной форме [100]:

$$
\begin{gathered}
\frac{\partial \boldsymbol{V}}{\partial t}+(\boldsymbol{V}, \nabla) \boldsymbol{V}=v \Delta \boldsymbol{V} ; \\
(\nabla, \boldsymbol{V})=0 .
\end{gathered}
$$


В уравнении Навье-Стокса (1) и уравнении несжимаемости (2) приняты обозначения: $\boldsymbol{V}=\left(V_{x}, V_{y}, V_{z}\right)$ - вектор скорости; $\boldsymbol{v}$ - кинематическая (молекулярная) вязкость жидкости; $\nabla=(\partial / \partial x, \partial / \partial y, \partial / \partial z)$ - оператор Гамильтона; $\Delta=\partial^{2} / \partial x^{2}+\partial^{2} / \partial y^{2}+\partial^{2} / \partial z^{2}-$ оператор Лапласа; круглые скобки в уравнении (1) определяют скалярное произведение.

Далее рассмотрим однонаправленные течения жидкости с полем скоростей:

$$
\boldsymbol{V}=\left(V_{x}(x, y, z, t), 0,0\right) \text {. }
$$
систему:

После подстановки вектора скорости (3) в уравнения (1) и (2) получим следующую

$$
\begin{gathered}
\frac{\partial V_{x}}{\partial t}=v\left(\frac{\partial^{2} V_{x}}{\partial x^{2}}+\frac{\partial^{2} V_{x}}{\partial y^{2}}+\frac{\partial^{2} V_{x}}{\partial z^{2}}\right) ; \\
\frac{\partial V_{x}}{\partial x}=0 .
\end{gathered}
$$

В последней системе уравнение (4) - параболическое уравнение типа теплопроводности размерности $(3+1)$. Из уравнения несжимаемости (5) следует, что скорости жидкости не зависят от координаты $x$. Таким образом, однонаправленное течение жидкости является неоднородным. Поле скоростей зависит от двух координат и времени:

$$
V_{x}=V_{x}(y, z, t) .
$$

Неизвестная функция скорости $V_{x}$, описывающая однонаправленное неоднородное изобарическое течение типа Куэтта, в этом случае удовлетворяет уравнению размерности $(2+1)$ :

$$
\frac{\partial V_{x}}{\partial t}=v\left(\frac{\partial^{2} V_{x}}{\partial y^{2}}+\frac{\partial^{2} V_{x}}{\partial z^{2}}\right)
$$

Далее приведены новые точные решения уравнения (7), которые также удовлетворяют уравнению Навье-Стокса (1) и уравнению несжимаемости (2). Заметим, что установившиеся течения жидкости $\boldsymbol{V}=\left(V_{x}(y, z), 0,0\right)$, как и классическое течение Куэтта, не зависят от вязкости:

$$
\frac{\partial^{2} V_{x}}{\partial y^{2}}+\frac{\partial^{2} V_{x}}{\partial z^{2}}=0
$$

Поскольку скорость $V_{x}$ удовлетворяет уравнению Лапласа, то эта функция является гармонической. 


\section{3. Класс точных решений}

Построение точного решения будет осуществляться при помощи метода разделенных переменных и его модификаций $[2 ; 3 ; 100]$. Отметим, что уравнению (7) удовлетворяет классическое точное решение Куэтта

$$
V_{x}=U(z, t),
$$

которое определяется из уравнения типа теплопроводности размерности $(1+1)$ :

$$
\frac{\partial U}{\partial t}=v \frac{\partial^{2} U}{\partial z^{2}}
$$

Если рассматривать установившееся течение $V_{x}=U(z)$ вязкой несжимаемой жидкости, то профиль скорости определяется из обыкновенного дифференциального уравнения второго порядка и имеет вид:

$$
U=C_{1} z+C_{2}
$$

Здесь $C_{1}$ и $C_{2}$ - постоянные интегрирования.

Другим известным частным решением уравнения (7) является следующее представление поля скорости:

$$
V_{x}=y u_{1}(z, t)
$$

В этом случае функция $u_{1}$ также удовлетворяет простейшему параболическому уравнению типа теплопроводности размерности $(1+1)$. Если учесть, что уравнение $(7)$ является линейным, то сумма частных решений (8) и (9)

$$
V_{x}=U(z, t)+y u_{1}(z, t)
$$

также является решением. Поставив выражение (10) в уравнение (7), получим уравнение:

$$
\frac{\partial U}{\partial t}+y \frac{\partial u_{1}}{\partial t}=v\left(\frac{\partial^{2} U}{\partial z^{2}}+y \frac{\partial^{2} u_{1}}{\partial z^{2}}\right) \text {. }
$$

Используя метод неопределенных коэффициентов относительно координаты $y$, получим систему уравнений, состоящую из двух изолированных уравнений параболического типа $(1+1)$, для определения скорости $U$ и пространственного ускорения $u_{1}$ :

$$
\frac{\partial U}{\partial t}=v \frac{\partial^{2} U}{\partial z^{2}} ; \frac{\partial u_{1}}{\partial t}=v \frac{\partial^{2} u_{1}}{\partial z^{2}}
$$


Рассмотрим сумму решения (10) и нелинейного слагаемого вида $\frac{y^{2}}{2} u_{2}(z, t)$ :

$$
V_{x}=U(z, t)+y u_{1}(z, t)+\frac{y^{2}}{2} u_{2}(z, t)
$$

Подставим сумму (11) в уравнение (7) и в результате несложных преобразований придем к следующему уравнению:

$$
\frac{\partial U}{\partial t}+y \frac{\partial u_{1}}{\partial t}+\frac{y^{2}}{2} \frac{\partial u_{2}}{\partial t}=v\left(u_{2}+\frac{\partial^{2} U}{\partial z^{2}}+y \frac{\partial^{2} u_{1}}{\partial z^{2}}+\frac{y^{2}}{2} \frac{\partial^{2} u_{2}}{\partial z^{2}}\right) .
$$

Это уравнение в силу метода неопределенных коэффициентов относительно координаты $y$ распадается на несколько уравнений типа теплопроводности размерности $(1+1)$ :

$$
\frac{\partial U}{\partial t}=v\left(u_{2}+\frac{\partial^{2} U}{\partial z^{2}}\right) ; \frac{\partial u_{1}}{\partial t}=v \frac{\partial^{2} u_{1}}{\partial z^{2}} ; \frac{\partial u_{2}}{\partial t}=v \frac{\partial^{2} u_{2}}{\partial z^{2}}
$$

Отметим, что в получившейся системе первое уравнение (уравнение для однородной скорости $U$ ) перестает быть изолированным. Этот факт объясняется именно нелинейностью последнего слагаемого решения (11) по переменной $y$. Другими словами, решение (11) уже не является суперпозицией ранее приведенных решений, т. е. наследует нелинейные свойства уравнений Навье-Стокса. Эта же тенденция сохраняется и при дальнейшем повышении степени для координаты $y$ в слагаемых, определяющих вид скорости $V_{x}$.

\section{4. Полиномиальные точные решения произвольного порядка}

Рассмотрим далее точное решение уравнения (7) в виде полиномиальных сумм специального вида:

$$
V_{x}=U(z, t)+\sum_{k=1}^{n} \frac{y^{k}}{k !} u_{k}(z, t)
$$

В формуле (13) $k$ ! обозначает взятие факториала от натурального числа $k$, коэффициенты $U$ и $u_{k}$ зависят от вертикальной координаты $z$ и времени $t$. Вид точного решения можно трактовать как применение модифицированного разделения переменных [101-108].

Посчитаем отдельно частные производные, необходимые для подстановки в уравнение типа теплопроводности (7):

$$
\begin{gathered}
\frac{\partial V_{x}}{\partial t}=\frac{\partial U}{\partial t}+\sum_{k=1}^{n} \frac{y^{k}}{k !} \frac{\partial u_{k}}{\partial t} \\
\frac{\partial^{2} V_{x}}{\partial z^{2}}=\frac{\partial^{2} U}{\partial z^{2}}+\sum_{k=1}^{n} \frac{y^{k}}{k !} \frac{\partial^{2} u_{k}}{\partial z^{2}}
\end{gathered}
$$




$$
\frac{\partial^{2} V_{x}}{\partial y^{2}}=\sum_{k=2}^{n} \frac{y^{k-2}}{(k-2) !} u_{k}
$$

Подставим получившиеся выражения в уравнение (7):

$$
\begin{gathered}
\frac{\partial U}{\partial t}+\sum_{k=1}^{n} \frac{y^{k}}{k !} \frac{\partial u_{k}}{\partial t}=v\left[\sum_{k=2}^{n} \frac{y^{k-2}}{(k-2) !} u_{k}+\frac{\partial^{2} U}{\partial z^{2}}+\sum_{k=1}^{n} \frac{y^{k}}{k !} \frac{\partial^{2} u_{k}}{\partial z^{2}}\right] \\
\frac{\partial U}{\partial t}+\sum_{k=1}^{n} \frac{1}{k !} \frac{\partial u_{k}}{\partial t} y^{k}=v\left[\frac{\partial^{2} U}{\partial z^{2}}+\sum_{k=2}^{n}\left(\frac{u_{k}}{(k-2) !}\right) y^{k-2}+\sum_{k=1}^{n} \frac{1}{k !} \frac{\partial^{2} u_{k}}{\partial z^{2}} y^{k}\right]
\end{gathered}
$$

Это уравнение можно переписать в виде системы дифференциальных уравнений, если применить метод неопределенных коэффициентов:

$$
\begin{gathered}
\frac{\partial U}{\partial t}=v\left(\frac{\partial^{2} U}{\partial z^{2}}+u_{2}\right) ; \\
\frac{\partial u_{1}}{\partial t}=v\left(u_{3}+\frac{\partial^{2} u_{1}}{\partial z^{2}}\right) ; \\
\frac{\partial u_{2}}{\partial t}=v\left(u_{4}+\frac{\partial^{2} u_{2}}{\partial z^{2}}\right) ; \\
\frac{\partial u_{n-2}}{\partial t}=v\left(u_{n}+\frac{\partial^{2} u_{n-2}}{\partial z^{2}}\right) ; \\
\frac{\partial u_{n-1}}{\partial t}=v \frac{\partial^{2} u_{n-1}}{\partial z^{2}} ; \\
\frac{\partial u_{n}}{\partial t}=v \frac{\partial^{2} u_{n}}{\partial z^{2}} .
\end{gathered}
$$

В получившейся системе только два изолированных уравнения (уравнения для коэффициентов $u_{n-1}$ и $\left.u_{n}(13)\right)$. Интегрирование системы (14) осуществляется в обратном порядке - от последнего уравнения к первому. Каждое из уравнений системы (14) является нестационарным уравнением типа уравнения теплопроводности. При этом бо́льшая часть указанных уравнений является неоднородными, неоднородность которых определяется в результате решения предыдущих уравнений. Таким образом, имеет место рекуррентный порядок интегрирования уравнений системы (14).

Заметим, что если в разложении (13) положить $n=2$, т. е. ограничиться только квадратичными членами, то система (14) примет вид:

$$
\frac{\partial U}{\partial t}=v\left(u_{2}+\frac{\partial^{2} U}{\partial z^{2}}\right) ; \frac{\partial u_{1}}{\partial t}=v \frac{\partial^{2} u_{1}}{\partial z^{2}} ; \frac{\partial u_{2}}{\partial t}=v \frac{\partial^{2} u_{2}}{\partial z^{2}} .
$$


Получившаяся система полностью совпадает с приведенной выше системой (12), определяющей компоненты решения (11).

Используем рекуррентный способ интегрирования для описания установившихся течений для поля скоростей (11), которое имеет вид:

$$
V_{x}=U(z)+y u_{1}(z)+\frac{y^{2}}{2} u_{2}(z) .
$$

В этом случае система уравнений в частных производных типа теплопроводности (12) редуцируется к системе обыкновенных дифференциальных уравнений шестого порядка:

$$
\frac{d^{2} U}{d z^{2}}=-u_{2} ; \frac{d^{2} u_{1}}{d z^{2}}=0 ; \frac{d^{2} u_{2}}{d z^{2}}=0
$$

Точное решение последней системы имеет вид:

$$
\begin{gathered}
u_{2}=C_{1} z+C_{2} ; u_{1}=C_{3} z+C_{4} ; \\
U=-\left(C_{1} \frac{z^{3}}{6}+C_{2} \frac{z^{2}}{2}\right)+C_{5} z+C_{6} .
\end{gathered}
$$

Последний коэффициент $u_{2}$ и предпоследний коэффициент $u_{1}$ являются линейными функциями, а однородное слагаемое $U$ описывается многочленом третьей степени.

Возвращаясь к исследованию поля скоростей (13), рассмотрим случай установившегося течения. Оно сводится к интегрированию системы уравнений (14) вида:

$$
\begin{gathered}
\frac{d^{2} U}{d z^{2}}=-u_{2} \\
\frac{d^{2} u_{1}}{d z^{2}}=-u_{3} \\
\frac{d^{2} u_{2}}{d z^{2}}=-u_{4} \\
\quad \ldots \\
\frac{d^{2} u_{n-2}}{d z^{2}}=-u_{n} \\
\frac{d^{2} u_{n-1}}{d z^{2}}=0 \\
\frac{d^{2} u_{n}}{d z^{2}}=0
\end{gathered}
$$

Интегрирование системы производится аналогично частному случаю для $n=2$. Коэффициенты $u_{n}$ и $u_{n-1}$ являются линейными функциями; коэффициенты, определяемые непосредственно через $u_{n}$ и $u_{n-1}$, - кубическими многочленами. Степень полиномов, описы- 
вающих остальные коэффициенты в представлении поля скорости, постепенно будет возрастать, каждый раз увеличиваясь на два.

\section{5. Завихренность и касательные напряжения}

Напомним, что для классического течения Куэтта касательное напряжение $\tau_{x z}=\eta \frac{\partial V_{x}}{\partial z}$ является постоянными, т. е. в жидкости регистрируются напряжения одного знака [1-12]. Вычислив касательное напряжение для поля скоростей (11), получим для него выражение:

$$
\tau_{x z}=\eta \frac{\partial V_{x}}{\partial z}=\eta\left(\frac{\partial U}{\partial z}+\sum_{k=1}^{n} \frac{\partial u_{k}}{\partial z} \frac{y^{k}}{k !}\right) .
$$

При учете пространственной неоднородности скорости однонаправленных течений поле касательного напряжения имеет сложную топологию зон растягивающих и сжимающих напряжений. Границы нулевого значения касательного напряжения $\tau_{x z}$ для каждого момента времени определяются алгебраическим уравнением:

$$
\frac{\partial U}{\partial z}+\sum_{k=1}^{n} \frac{\partial u_{k}}{\partial z} \frac{y^{k}}{k !}=0 .
$$

Неоднородное распределение скорости влечет появление в жидкости касательного напряжения:

$$
\tau_{x y}=\eta \frac{\partial V_{x}}{\partial y}=\eta \sum_{k=1}^{n} u_{k} \frac{y^{k-1}}{(k-1) !} .
$$

Проанализируем величину завихренности потока для точного решения вида (13). Согласно определению, завихренность определяется следующим определителем:

$$
\begin{gathered}
\boldsymbol{\Omega}=\left|\begin{array}{ccc}
\boldsymbol{i} & \boldsymbol{j} & \boldsymbol{k} \\
\frac{\partial}{\partial x} & \frac{\partial}{\partial y} & \frac{\partial}{\partial z} \\
V_{x} & V_{y} & V_{z}
\end{array}\right|=\left|\begin{array}{ccc}
\boldsymbol{i} & \boldsymbol{j} & \boldsymbol{k} \\
\frac{\partial}{\partial x} & \frac{\partial}{\partial y} & \frac{\partial}{\partial z} \\
V_{x} & 0 & 0
\end{array}\right|=\frac{\partial V_{x}}{\partial z} \boldsymbol{j}-\frac{\partial V_{x}}{\partial y} \boldsymbol{k}= \\
=\left(\frac{\partial U}{\partial z}+\sum_{k=1}^{n} \frac{\partial u_{k}}{\partial z} \frac{y^{k}}{k !}\right) \boldsymbol{j}-\left(\sum_{k=1}^{n} u_{k} \frac{y^{k-1}}{(k-1) !}\right) \boldsymbol{k} .
\end{gathered}
$$

Учитывая выражения для касательных напряжений, получим следующее разложение вектора завихренности:

$$
\boldsymbol{\Omega}=\frac{\tau_{x z}}{\eta} \boldsymbol{j}-\frac{\tau_{x y}}{\eta} \boldsymbol{k}
$$

На основании полученного выражения можно утверждать, что рассматриваемое однонаправленное течение вида (13) является всюду (возможно, за исключением нескольких точек) вихревым. Зоны потенциального течения определяются условием: 


$$
\tau_{x z}^{2}+\tau_{x y}^{2}=0
$$

При этом возникновение завихренности потока объясняется сильной нелинейной зависимостью структуры решения (13) от горизонтальной координаты $y$.

\section{6. Заключение}

В статье предложен новый класс решений уравнений Навье-Стокса для описания нестационарного изобарического однонаправленного вертикально завихренного течения жидкости. Отличительной особенностью указанного класса является полиномиальная зависимость произвольного порядка скорости от одной из горизонтальных координат, причем коэффициенты данного полиномиального представления зависят произвольным образом как от вертикальной координаты, так и от времени. В статье доказано, что решения такого вида, начиная с полиномов второго порядка, не могут быть получены суперпозицией решений меньшей степени. Приведена система уравнений, позволяющая определить переменные коэффициенты в представлении скорости, решение системы сводится к последовательному интегрированию нестационарных неоднородных уравнений типа теплопроводности.

\section{Литература}

1. Aristov S. N., Knyazev D. V., Polyanin A. D. Exact solutions of the Navier-Stokes equations with the linear dependence of velocity components on two space variables // Theoretical Foundations of Chemical Engineering. - 2009. - Vol. 43, No. 5. - P. 642-662. DOI: $10.1134 / \mathrm{S} 0040579509050066$.

2. Drazin P. G. and Riley N. The Navier-Stokes Equations: A classification of flows and exact solutions. - Cambridge : Cambridge Univ. Press, 2006. - 196 p.

3. Polyanin A. D., Zaitsev V. F. Handbook of nonlinear partial differential equations. - Boca Raton : Chapman \& Hall / CRC Press, 2004. - 840 p.

4. Whitham G. B. The Navier-Stokes equations of motion / ed. L. Rosenhead. - Oxford, Clarendon : Laminar Boundary Layers, 1963. - P. 114-162.

5. Dryden H. L., Murnaghan F. D., Bateman H. Report of the committee on hydrodynamics // Bull. Natl. Res. Counc. (US). - 1932. - Vol. 84 - P. 155-332.

6. Berker R. Sur quelques cas d'Integration des equations du mouvement d'un fuide visquex incomprcssible. - Paris-Lille: Taffin-Lefort, 1936.

7. Berker R. Integration des equations du mouvement d'un fluide visqueux incompressible / ed. S. Flugge. - Berlin : Springer-Verlag. Handbuch der Physik, 1963. - VIII/2. - 384 p.

8. Wang C. Y. Exact solution of the Navier-Stokes equations-the generalized Beltrami flows, review and extension // Acta Mech. - 1990. - Vol. 81. - P. 69-74. - DOI: 10.1007/BF01174556.

9. Wang C. Y. Exact solutions of the steady-state Navier-Stokes equations // Annu. Rev. Fluid Mech. - 1991. - Vol. 23. - P. 159-177. - DOI: 10.1146/annurev.fl.23.010191.001111.

10. Wang, C. Y. Exact solutions of the unsteady Navier-Stokes equations // Appl. Mech. Rev. 1989. - Vol. 42 (11S). - P. 269-282. - DOI: 10.1115/1.3152400.

11. Пухначев В. В. Симметрии в уравнениях Навье-Стокса // Успехи механики. - 2006. № 1. - C. 6-76.

12. Couette M. Etudes sur le frottement des liquids // Ann. Chim. Phys. - 1890. - Vol. 21. P. 433-510.

13. Stokes G. G. On the effect of the internal friction of fluid on the motion of pendulums // Camb. Philo. Trans. - 1851. - Vol. 9. - P. 8-106. 
14. Taylor G. I. Stability of a Viscous Liquid Contained between Two Rotating Cylinders // Philosophical Transactions Royal Society of London. - 1923. - Vol. 223, No. 605-615. P. 289-343. - DOI: 10.1098/rsta.1923.0008.

15. Holodniok M., Kubíček M., Hlaváček V. Computation of the flow between two rotating coaxial disk: multiplicity of steady-state solutions // J. Fluid Mech. - 1981. - Vol. 108. P. 227-240. - DOI: 10.1017/S0022112081002097.

16. Aristov S. N., Gitman I. M. Viscous flow between two moving parallel disks. Exact solutions and stability analysis // J. Fluid Mech. - 2002. - Vol. 464. - P. 209-215. DOI: $10.1017 / \mathrm{S} 0022112002001003$.

17. Zhilenko D. Y., Krivonosova O. E. Transitions to chaos in the spherical Couette flow due to periodic variations in the rotation velocity of one of the boundaries // Fluid Dynamics. - 2013. Vol. 48, No. 4. - P. 452-460. - DOI: 10.1134/S0015462813040042.

18. Wave number selection in the presence of noise: Experimental results / D. Zhilenko, O. Krivonosova, M. Gritsevich, P. Read // Chaos. - 2018. - Vol. 28. - 053110. - DOI: 10.1063/1.5011349.

19. Zhilenko D. Y., Krivonosova O. E. Origination and evolution of turbulent flows in a rotating spherical layer // Technical Physics. - 2010. - Vol. 55, No. 4. - P. 449-456. DOI: $10.1134 / \mathrm{S} 1063784210040031$.

20. Беляев Ю. Н., Монахов А. А., Яворская И. М. Устойчивость сферического течения Куэтта в толстых слоях при вращении внутренней сферы // Известия Академии наук СССР. Механика жидкости и газа. - 1978. - № 2. - С. 9-15.

21. Pukhnachev V. V., Pukhnacheva T. P. The Couette problem for a Kelvin-Voigt medium // J. Math. Sci. - 2012. - Vol. 186. - P. 495-510. - DOI: 10.1007/s10958-012-1003-0.

22. Zhuk V. I., Protsenko I. G. Asymptotic model for the evolution of perturbations in the plane Couette-Poiseuille flow // Doklady Mathematics. - 2006. - Vol. 74, No. 3. - P. 896-900. DOI: $10.1134 / \mathrm{S} 1064562406060287$.

23. Gavrilenko S. L., Shil'ko S. V., Vasin R. A. Characteristics of a viscoplastic material in the Couette flow // Journal of Applied Mechanics and Technical Physics. - 2002. - Vol. 43, No. 3. P. 439-444. - DOI: 10.1023/A:1015378622918.

24. Troshkin O. V. Nonlinear stability of Couette, Poiseuille, and Kolmogorov plane channel flows // Dokl. Math. - 2012. - Vol. 85. - P. 181-185. - DOI: 10.1134/S1064562412020068.

25. Rudyak V., Isakov E., \& Bord E. Instability of plane Couette flow of two-phase liquids // Tech. Phys. Lett. - 1998. - Vol. 24. - P. 199-200. - DOI: 10.1134/1.1262051.

26. Shalybkov D. A. Hydrodynamic and hydromagnetic stability of the Couette flow // PhysicsUspekhi. - 2009. - Vol. 52, No. 9. - P. 915-935. - DOI: 10.3367/UFNe.0179.200909d.0971.

27. Boronin S. A. Stability of the plane Couette flow of a disperse medium with a finite volume fraction of the particles // Fluid Dynamics. - 2011. - Vol. 46. - P. 64-71. DOI: $10.1134 / \mathrm{S} 0015462811010078$.

28. Кудинов В. А., Кудинов И. В. Получение точных аналитических решений гиперболических уравнений движения при разгонном течении Куэтта // Изв. РАН. Энергетика. 2012. - № 1. - C. 119-133.

29. Babkin V. A. Plane Turbulent Couette Flow // Journal of Engineering Physics and Thermophysics. - 2003. - Vol. 76. - P. 1251-1254. - DOI: 10.1023/B:JOEP.0000012026.19646.c6.

30. Абрамян А. К., Миранцев Л. В., Кучмин А. Ю. Моделирование течения Куэтта простой жидкости в плоском канале наноразмерной высоты // Математическое моделирование. 2012. - T. 24, № 4. - C. 3-21.

31. Malyshev V. and Manita A. Stochastic Micromodel of the Couette Flow // Theor. Prob. Appl. - 2009. - Vol. 53, No. 4. - P. 716-727. - DOI: 10.1137/S0040585X97983924.

32. Georgievskii D. V. Generalized Joseph estimates of stability of plane shear flows with scalar nonlinearity // Bull. Russ. Acad. Sci. Phys. - 2011 - Vol. 75. - P. 140-143. DOI: $10.3103 / \mathrm{S} 1062873810121044$. 
33. Беляева Н. А., Кузнецов К. П. Анализ нелинейной динамической модели течения Куэтта структурированной жидкости в плоском зазоре // Вестник СамГТУ. Сер. Физикоматематические науки. - 2012. - № 2 (27). - С. 85-92.

34. Boundary slip in Newtonian liquids: a review of experimental studies / C. Neto, D. Evans, E. Bonaccurso, H.-J. Butt, V. S. J. Craig // Rep. Prog. Phys. - 2005. - Vol. 68. - P. 2859-2897. DOI: $10.1088 / 0034-4885 / 68 / 12 / \mathrm{R} 05$.

35. Beirao da Veiga H. Regularity for Stokes and general-ized Stokes systems under nonhomogeneous slip-typeboundary conditions // Adv. Different. - 2004. - 9 (9-10). - P. 1079-1114.

36. Bocquet L., Charlaix E. Nanofluidics, from bulk to interfaces // Chem. Soc. Rev. - 2010. Vol. 39. - P. 1073-1095. - DOI: 10.1039/b909366b.

37. Bouzigues C. I., Tabeling P., Bocquet L. Nanofluidics in the Debye Layer at Hydrophilic and Hydrophobic Surfaces // Phys. Rev. Lett. - 2008. - Vol. 101. - P. 114503.

38. Ng C. O., Wang C. Y. Apparent slip arising from Stokes shear flow over a bidimensional patterned surface // Microfluid Nanofluid. - 2010. - Vol. 8. - P. 361-371. - DOI: 10.1007/s10404009-0466-x.

39. Wang Y., Bhushan B. Boundary slip and nanobubble study in micro/nanofluidics using atomic force microscopy // Soft Matter. - 2010. - Vol. 6. - P. 29-66. - DOI: 10.1039/B917017K.

40. Schwarz K. G., Schwarz Y. A. Stability of Advective Flow in a Horizontal Incompressible Fluid Layer in the Presence of the Navier Slip Condition // Fluid Dynamics. - 2020. - Iss. 55. P. 31-42. - DOI: 10.1134/S0015462820010115.

41. Burmasheva N. V., Privalova V. V., Prosviryakov E. Y. Layered Marangoni convection with the Navier slip condition // Sādhanā. - 2021. - Vol. 46. - 55. - DOI: 10.1007/s12046-02101585-5.

42. Привалова В. В., Просвиряков Е. Ю. Нелинейное изобарическое течение вязкой несжимаемой жидкости в тонком слое с проницаемыми границами // Вычислительная механика сплошных сред. - 2019. - Т. 12, № 2. - C. 230-242. - DOI: 10.7242/1999-6691/2019.12.2.20.

43. Birikh R. V. Thermocapillary convection in a horizontal layer of liquid // J. Appl. Mech. Tech. Phys. - 1966. - No. 7. - P. 43-44. - DOI: 10.1007/BF00914697.

44. Остроумов Г. А. Свободная конвекция в условиях внутренней задачи. - М. : Гостехиздат, 1952. $-256 \mathrm{c}$.

45. Smith M. K., Davis S. H. Instabilities of dynamic thermocapillary liquid layers. Part. 1. Convective instabilities // J. Fluid Mech. - 1983. - Vol. 132. - P. 119-144. DOI: $10.1017 / \mathrm{S} 0022112083001512$.

46. Ortiz-Pérez A. S., Dávalos-Orozco L. A. Convection in a horizontal fluid layer under an inclined temperature gradient // Phys. Fluid. - 2011. - Vol. 23. - 084107. - DOI: 10.1063/1.3626009.

47. Бурмашева Н. В., Просвиряков Е. Ю. Точное решение для установившихся конвективных концентрационных течений типа Куэтта // Вычислительная механика сплошных сред. - 2020. - Т. 13, № 3. - С. 337-349. - DOI: 10.7242/1999-6691/2020.13.3.27.

48. Аристов С. Н., Шварц К. Г. Вихревые течения адвективной природы во вращающемся слое жидкости. - Пермь : Изд-во Пермск. гос. ун-та, 2006.

49. Aristov S. N., Prosviryakov E. Y. A new class of exact solutions for three-dimensional thermal diffusion equations // Theoretical Foundations of Chemical Engineering. - 2016. - Vol. 50, No. 3. - P. 286-293. - DOI: 10.1134/S0040579516030027.

50. Burmasheva N. V., Prosviryakov E. Y. Thermocapillary convection of a vertical swirling liquid // Theoretical Foundations of Chemical Engineering. - 2020. - Vol. 54, No. 1. - P. 230-239. DOI: $10.1134 /$ S0040579519060034.

51. Birikh R. V., Pukhnachev V. V., Frolovskaya O. A. Convective flow in a horizontal channel with non-newtonian surface rheology under time-dependent longitudinal temperature gradient // Fluid Dynamics. - 2015. - Vol. 50, No. 1. - P. 173-179. - DOI: 10.1134/S0015462815010172. 
52. Бурмашева Н.В., Просвиряков Е.Ю. Крупномасштабная слоистая стационарная конвекция вязкой несжимаемой жидкости под действием касательных напряжений на верхней границе. Исследование поля скоростей // Вестн. Сам. гос. техн. ун-та. Сер. Физ.-мат. науки. 2017. - T. 21, вып. 1. - C. 180-196. - DOI: 10.14498/vsgtu1527.

53. Бурмашева Н. В., Просвиряков Е. Ю. Крупномасштабная слоистая стационарная конвекция вязкой несжимаемой жидкости под действием касательных напряжений на верхней границе. Исследование полей температуры и давления // Вестн. Сам. гос. техн. ун-та. Cер. Физ.-мат. науки. - 2017. - Т. 21, вып. 4. - C. 736-751. - DOI: 10.14498/vsgtu1568.

54. Burmasheva N. V., Prosviryakov E. Yu. Convective layered flows of a vertically whirling viscous incompressible fluid. Velocity field investigation // Вестник СамГТУ. Сер. Физ.-мат. науки. - 2019. - Т. 23, вып. 2. - С. 341-360. - DOI: 10.14498/vsgtu1670.

55. Аристов С. Н., Привалова В. В., Просвиряков Е. Ю. Стационарное неизотермическое течение Куэтта. Квадратичный нагрев верхней границы слоя жидкости // Нелинейная динамика. - 2016. - Т. 12, вып. 2. - С. 167-178. - DOI: 10.20537/nd1602001.

56. Burmasheva N. V., Prosviryakov E. Yu. On Marangoni shear convective flows of inhomogeneous viscousincompressible fluids in view of the Soret effect // Journal of King Saud University Science. - 2020. - Vol. 32, iss. 8. - P. 3364-3371. - DOI: 10.1016/j.jksus.2020.09.023.

57. Schwarz K. G. Plane-parallel advective flow in a horizontal incompressible fluid layer with rigid boundaries // Fluid Dynamics. - 2019. - Vol. 49, No. 4. - P. 438-442. DOI: $10.1134 / \mathrm{S} 0015462819110016$.

58. Andreev V. K., Stepanova I. V. Unidirectional flows of binary mixtures within the framework of the Oberbeck-Boussinesq model // Fluid Dynamics. - 2016. - Vol. 51, No. 2. - P. 136-147. DOI: $10.1134 / \mathrm{S} 0015462816020022$.

59. Andreev V. K., Stepanova I. V. Ostroumov-Birikh solution of convection equations with nonlinear buoyancy force // Appl. Math. Comput. - 2014. - Vol. 228. - P. 59-67. DOI: $10.1016 /$ j.amc.2013.11.002.

60. Bekezhanova V. B. Change of the types of instability of a steady two-layer flow in an inclined channel // Fluid Dynamics. - 2011. - Vol. 46 (525). - DOI: 10.1134/S001546281104003X.

61. Горшков А. В., Просвиряков Е. Ю. Конвективное слоистое течение Экмана вязкой несжимаемой жидкости // Известия РАН. Физика атмосферы и океана. - 2018. - Т. 54, вып. 2. - С. 213-220. - DOI: 10.7868/S0003351518020101.

62. Пухначев В. В. Нестационарные аналоги решения Бириха // Изв. АлтГУ. - 2011. № 1-2 (69). - C. 62-69.

63. Shvarz K. G. Plane-parallel advective flow in a horizontal incompressible fluid layer with rigid boundaries // Fluid Dynamics. - 2014. - Vol. 49, No. 4. - P. 438-442. DOI: $10.1134 / \mathrm{S} 0015462814040036$.

64. Андреев В. К. Решения Бириха уравнений конвекции и некоторые его обобщения : Препринт. - Красноярск : ИВМ СО РАН, 2010. - 24 с.

65. Aristov S. N., Prosviryakov E. Y., Spevak L. F. Unsteady-state Bénard-Marangoni convection in layered viscous incompressible flows // Theoretical Foundations of Chemical Engineering. 2016. - Vol. 50, No. 2. - P. 132-141. - DOI: 10.1134/S0040579516020019.

66. Аристов С. Н., Просвиряков Е. Ю., Спевак Л. Ф. Нестационарная слоистая тепловая и концентрационная конвекция Марангони вязкой жидкости // Вычисл. мех. сплош. сред. 2015. - T. 8, № 4. - C. 445-456. - DOI: 10.7242/1999-6691/2015.8.4.38.

67. Аристов С.Н., Просвиряков Е.Ю. О слоистых течениях плоской свободной конвекции // Нелин. динам. - 2013. - Т. 9. - № 4. - С. 651-657.

68. Горшков А. В., Просвиряков Е. Ю. Слоистая конвекция Бенара-Марангони при теплообмене по закону Ньютона-Рихмана // Компьютерные исследования и моделирование. 2016. - Т. 8, № 6. - C. 927-940. 
69. Горшков А. В., Просвиряков Е. Ю. Аналитические решения стационарной сложной конвекции, описывающие поле касательных напряжений разного знака // Тр. ИММ УрО PAH. - 2017. - T. 23, № 2. - C. 32-41. - DOI: 10.21538/0134-4889-2017-23-2-32-41.

70. Knyazev D. V. Two-dimensional flows of a viscous binary fluid between moving solid boundaries // Journal of Applied Mechanics and Technical Physics. - 2011. - Vol. 52, No. 2. P. 212-217. - DOI: 10.1134/S0021894411020088.

71. Компаниец Л. А., Питальская О. С. Точные решения модели Экмана трехмерного ветрового движения однородной жидкости с учетом геострофической составляющей // Компьютерные исследования и моделирование. - 2009. - Т. 1, вып. 1. - С. 57-66. DOI: 10.20537/2076-7633-2009-1-1-57-66.

72. Бурмашева Н. В., Просвиряков Е. Ю. Точное решение уравнений Навье-Стокса, описывающее пространственно неоднородные течения вращающейся жидкости // Труды Института математики и механики УрО РАН. - 2020. - Т. 26. Вып. 2. - С. 79-87. DOI: $10.21538 / 0134-4889-2020-26-2-79-87$.

73. Бурмашева Н. В., Просвиряков Е. Ю. Класс точных решений для двумерных уравнений геофизической гидродинамики с двумя параметрами Кориолиса // Известия Иркутского государственного университета. Серия Математика. - 2020. - Т. 32. - С. 33-48. DOI: $10.26516 / 1997-7670.2020 .32 .33$.

74. Burmasheva N. V., Prosviryakov E. Yu. An exact solution for describing the unidirectional Marangoni flow of a viscous incompressible fluid with the Navier boundary condition. Temperature field investigation // Diagnostics, Resource and Mechanics of materials and structures. - 2020. Iss. 1. - P. 6-23. - DOI: 10.17804/2410-9908.2020.1.006-023 . - URL: https://dreamjournal.org/issues/2020-1/2020-1_278.html

75. Burmasheva N. V. and Prosviryakov E. Yu. Exact solution for describing a unidirectional Marangoni flow of a viscous incompressible fluid with the Navier boundary condition. Pressure field investigation // Diagnostics, Resource and Mechanics of materials and structures. - 2020. Iss. 2. - P. 61-75. - DOI: 10.17804/2410-9908.2020.2.061-075. - URL: https://dreamjournal.org/DREAM_Issue_2_2020_Burmasheva_N.V._et_al._061_075.pdf

76. Lin C. C. Note on a class of exact solutions in magneto-hydrodynamics // Arch. Rational Mech. Anal. - 1957. - Vol. 1. - P. 391-395. - DOI: 10.1007/BF00298016.

77. Sidorov A. F. Two classes of solutions of the fluid and gas mechanics equations and their connection to traveling wave theory // J. Appl. Mech. Tech. Phys. - 1989. - Vol. 30, No. 2. P. 197-203. - DOI: 10.1007/BF00852164.

78. Аристов С. Н. Вихревые течения в тонких слоях жидкости : автореф. дис. ... докт. физ.-мат. наук: 01.02.05 / Аристов Сергей Николаевич. - Владивосток : ИАПУ, 1990. - 303 с.

79. Prosviryakov E. Y. New class of exact solutions of navier-stokes equations with exponential dependence of velocity on two spatial coordinates // Theoretical Foundations of Chemical Engineering. - 2019. - Vol. 53, No. 1. - P. 107-114. - DOI: 10.1134/S0040579518060088.

80. Аристов С. Н., Просвиряков Е. Ю. Крупномасштабные течения завихренной вязкой несжимаемой жидкости // Известия высших учебных заведений. Авиационная техника. 2015. - Вып. 4. - С. 50-54.

81. Аристов С. Н., Просвиряков Е. Ю. Неоднородные течения Куэтта // Нелинейная динамика. - 2014. - Т. 10, вып. 2. - C. 177-182. - DOI: 10.20537/nd1402004.

82. Privalova V. V., Prosviryakov E. Yu. Vortex flows of a viscous incompressible fluid at constant vertical velocity under perfect slip conditions // Diagnostics, Resource and Mechanics of materials and structures. - 2019. - Iss. 2. - P. 57-70. - DOI: 10.17804/2410-9908.2019.2.057-070. URL: https://dream-journal.org/issues/2019-2/2019-2_249.html

83. Aristov S. N., Prosviryakov E. Y. Unsteady layered vortical fluid flows // Fluid Dynamics. 2016. - Vol. 51, No. 2. - P. 148-154. - DOI: 10.1134/S0015462816020034. 
84. Zubarev N. M., Prosviryakov E. Y. Exact solutions for layered three-dimensional nonstationary isobaric flows of a viscous incompressible fluid // Journal of Applied Mechanics and Technical Physics. 2019. - Vol. 60, No. 6. - P. 1031-1037. - DOI: 10.1134/S0021894419060075.

85. Просвиряков Е. Ю. Точные решения трехмерных потенциальных и завихренных течений Куэтта вязкой несжимаемой жидкости // Вестник Национального исследовательского ядерного университета МИФИ. - 2015. - Т. 4, вып. 6. - С. 501-506. - DOI: 10.1134/S2304487X15060127.

86. Privalova V. V., Prosviryakov E. Yu., Simonov M. A. Nonlinear gradient flow of a vertical vortex fluid in a thin layer // Нелинейн. динам. - 2019. - Т. 15, вып. 3. - С. 271-283. DOI: $10.20537 / \mathrm{nd} 190306$.

87. Аристов С. Н., Просвиряков Е. Ю. Неоднородное конвективное течение Куэтта // Известия Российской академии наук. Механика жидкости и газа. - 2016. - № 5. - С. 3-9. DOI: $10.7868 / \mathrm{S} 0568528116050030$.

88. Privalova V.V., Prosviryakov E.Yu. The effect of tangential boundary stresses on the convective unidirectional flow of viscous fluid layer under the heating the lower boundary condition // Diagnostics, Resource and Mechanics of materials and structures. - 2019. - Iss. 4. - P. 44-55. DOI: 10.17804/2410-9908.2019.4.044-055. - URL: https://dream-journal.org/issues/2019-4/20194_262.html

89. Privalova V. V., Prosviryakov E. Yu. Exact solutions for the convective creep Couette-Hiemenz flow with the linear temperature distribution on the upper border // Diagnostics, Resource and Mechanics of materials and structures. - 2018. - Iss. 2. - P. 92-109. - DOI: 10.17804/2410-9908.2018.2.092-109. URL: https://dream-journal.org/DREAM_Issue_2_2018_Privalova_V.V._et_al._092_109.pdf

90. Аристов С. Н., Привалова В. В., Просвиряков Е. Ю. Плоская линейная конвекция Бенара-Рэлея при квадратичном нагреве верхней границы слоя вязкой несжимаемой жидкости // Вестник Казанского государственного технического университета им. А.Н. Туполева. 2015. - Т. 71, № 2. - С. 69-75.

91. Привалова В. В., Просвиряков Е. Ю. Стационарное конвективное течение Куэтта при линейном нагреве нижней границы слоя жидкости // Вестник Казанского государственного технического университета им. А.Н. Туполева. - 2015. - Т. 71, № 5. - С. 148-153.

92. Aristov S. N., Privalova V. V., Prosviryakov E. Y. Stationary nonisothermal Couette flow. Quadratic heating of the upper boundary of the fluid layer // Russian Journal of Nonlinear Dynamics. - 2016. - Vol. 12, No. 2. - P. 167-178. - DOI: 10.20537/nd1602001.

93. Аристов С. Н., Просвиряков Е. Ю. Об одном классе аналитических решений стационарной осесимметричной конвекции Бенара-Марангони вязкой несжимаемой жидкости // Вестн. Сам. гос. техн. ун-та. Сер. Физ.-мат. науки. - 2013. - № 3 (32). - С. 110-118.

94. Аристов С. Н., Просвиряков Е. Ю. Точные решения термокапиллярной конвекции при локализованном нагреве плоского слоя вязкой несжимаемой жидкости // Вестн. Казан. гос. техн. ун-та им. А. Н. Туполева. - 2014. - № 3. - С. 7-12.

95. Andreev V. K., Cheremnykh E. N. 2D thermocapillary motion of three fluids in a flat chan_nel. Journal of Siberian Federal University // Mathematics and Physics. - 2016. - Vol. 9, iss. 4. - P. 404-415. - DOI: 10.17516/1997-1397-2016-9-4-404-415.

96. Andreev V. K., Cheremnykh E. N. The joint creeping motion of three viscid liquids in a plane layer: A priori estimates and convergence to steady flow // Journal of Applied and Industrial Mathematics. - 2016. - Vol. 10, No. 1. - P. 7-20. - DOI: 10.1134/S1990478916010026.

97. Андреев В. К., Собачкина Н. Л. Движение бинарной смеси в плоских и цилиндриче_ских областях. - Красноярск : СФУ, 2012. - 187 с.

98. Hiemenz K. Die Grenzschicht an einem in den gleichförmigen Flüssigkeit-sstrom eingetauchten geraden Kreiszylinder // Dingler's Politech. J. - 1911. - Vol. 326. - P. 321-324.

99. Riabouchinsky D. Quelques considerations sur les mouvements plans rotationnels d' un liquide // C. R. Hebdomadaires Acad. Sci. - 1924. - Vol. 179. - P. 1133-1136.

100. Ландау Л. Д., Лифшиц Е. М. Гидродинамика. - 6-е. изд. - М. : Физматлит, 2006. 
101. Полянин А. Д., Журов А. И. Методы разделения переменных и точные решения нелинейных уравнений математической физики. - М. : Изд-во «ИПМех РАН», 2020. - 384 с.

102. Aristov S. N., Polyanin A. D. Exact solutions of unsteady three-dimensional NavierStokes equations // Doklady Physics. - 2009. - Vol. 54, No. 7. - P. 316-321. DOI: $10.1134 / \mathrm{S} 1028335809070039$.

103. Полянин А. Д. Точные решения уравнений Навье-Стокса с обобщенным разделением переменных // Доклады АН. - 2001. - Т. 380, № 4. - С. 491-496.

104. Полянин А. Д. Методы функционального разделения переменных и их применение в математической физике // Мат. моделирование и численные методы. - 2019. - № 1. C. 65-97. - DOI: 10.18698/2309-3684-2019-1-6597.

105. Polyanin A. D., Aristov S. N. Systems of hydrodynamic type equations: exact solutions, transformations, and nonlinear stability // Doklady Physics. - 2009. - Vol. 54, No. 9. - P. 429-434. DOI: $10.1134 / \mathrm{S} 1028335809090079$.

106. Полянин А. Д., Журов А. И. Решения с функциональным разделением переменных двух классов нелинейных уравнений математической физики // Докл. АН. - 2019. - Т. 486, № 3. - C. 287-291. - DOI: 10.31857/S0869-56524863287-291.

107. Aristov S. N., Polyanin A. D. New classes of exact solutions and some transformations of the Navier-Stokes equations // Russian J. Math. Physics. - 2010. - Vol. 17, No. 1. - P. 1-18. DOI: $10.1134 / \mathrm{S} 1061920810010012$.

108. Meleshko S. V. A particular class of partially invariant solutions of the NavierStokes equations // Nonlinear Dynamics. - 2004. - Vol. 36, No. 1. - P. 47-68. DOI: 10.1023/B:NODY.0000034646.18621.73. 